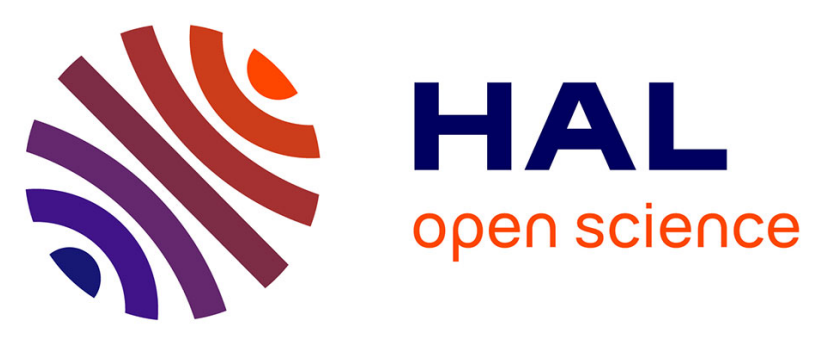

\title{
Computing Microstructures
}

\author{
M. Chipot
}

\section{To cite this version:}

M. Chipot. Computing Microstructures. Journal de Physique IV Proceedings, 1996, 06 (C1), pp.C127-C1-34. 10.1051/jp4:1996103 . jpa-00254134

\section{HAL Id: jpa-00254134 https://hal.science/jpa-00254134}

Submitted on 1 Jan 1996

HAL is a multi-disciplinary open access archive for the deposit and dissemination of scientific research documents, whether they are published or not. The documents may come from teaching and research institutions in France or abroad, or from public or private research centers.
L'archive ouverte pluridisciplinaire HAL, est destinée au dépôt et à la diffusion de documents scientifiques de niveau recherche, publiés ou non, émanant des établissements d'enseignement et de recherche français ou étrangers, des laboratoires publics ou privés. 


\title{
Computing Microstructures
}

\author{
M. Chipot
}

Centre d'Analyse Non Linéaire, Université de Metz, Ile du Saulcy, 57045 Metz cedex 01, France

\begin{abstract}
Minimization of energies with no minimizer produces minimizing sequences that could converge toward zero even though their gradients do not and oscillate. We analyse here this phenomenon in particular from the numerical point of view describing the problems faced in computing the corresponding microstructures.
\end{abstract}

\section{INTRODUCTION}

We denote by $\Omega$ a polygonal domain of $\mathbf{R}^{n}$. If $M^{m \times n}$ is the space of $m \times n$ matrices, let

$$
\varphi: M^{m \times n} \rightarrow \mathbf{R}^{+}
$$

be a continuous function. In various physical problems one is led to minimize energies of the type

$$
\int_{\Omega} \varphi(\nabla v(x)) d x
$$

over some class of vector valued functions $v=\left(v^{1}, \cdots, v^{m}\right) . \nabla v \in M^{m \times n}$ denotes here the jacobian matrix of $v$ whose entries are $\partial v^{i} / \partial x^{j}$. Now, to model states of low energy one assumes that the energy density $\varphi$ satisfies

$$
\varphi \geq 0, \varphi\left(W_{1}\right)=\varphi\left(W_{2}\right)=\cdots=\varphi\left(W_{k}\right)=0
$$

where $W_{i}$ are matrices or "wells" in $M^{m \times n}$.

As one can show, these problems do not admit in general minimizers. So, one is led to study their minimizing sequences and to analyse their relevance in describing physical phenomena. One crucial step toward the explanation of the physics of the problem at hand could be to discover a commun pattern for these minimizing sequences. If this is theoretically possible for some particular cases it is an other matter to drive the computer to find systematically these right patterns. Indeed, the functionals 
that we are led to study, and more especially their approximations, experience several local minima which all become traps for our codes. Our efforts lately have been devoted to try to understand how to become more successful in this respect. Of course one expects that many physical systems could be described by energies given by (1.1)-(1.3). Nonlinear elasticity enters this framework in the case $m=n=3$. Conditions like (1.3) have been introduced by several authors (see [2], [3], [4]-[14], [17], [18], [20], [21], [23], [24]). Let us quote few examples that have been investigated lately :

Examples : 1) $m=1, n=2$. If $W_{0}^{1, \infty}(\Omega, \mathbf{R})$ is the set of Lipschitz continuous functions with values in $\mathbf{R}$, vanishing on the boundary of $\Omega$ (see [19] for information on these spaces), consider

$$
\inf _{W_{0}^{1, \infty}(\Omega, \mathbf{R})} \int_{\Omega} v_{x}^{2}+\left(v_{y}^{2}-1\right)^{2} d x d y .
$$

$\left(v_{x}, v_{y}\right.$ denote respectively $\left.\partial v / \partial x, \partial v / \partial y\right)$. In this case the function $\varphi$ has two wells namely

$$
W_{1}=(0,1) \quad, \quad W_{2}=(0,-1) .
$$

(See [24] for the link with austenite-martensite transformation).

2) $m=n=2$. To study the deformation of a two dimensional crystal (see [18]) J. Ericksen and $\mathrm{R}$. James introduced the energy density

$$
\phi(F)=\tilde{\phi}(C)=\kappa_{1}(\operatorname{tr}(C)-2)^{2}+\kappa_{2} c_{12}^{2}+\kappa_{3}\left(\left(\frac{c_{11}-c_{22}}{2}\right)^{2}-\epsilon^{2}\right)^{2}
$$

where

$$
C=F^{T} F=\left(\begin{array}{ll}
c_{11} & c_{12} \\
c_{21} & c_{22}
\end{array}\right)
$$

is the Cauchy-Green strain tensor, $\kappa_{i}$ elastic moduli and $\epsilon$ the transformation strain. In this case the funtion $\varphi=\phi$ is frame invariant which leads to two "infinite" wells i.e. $\omega_{i}=\left\{Q W_{i} \mid Q \in S O_{2}\right\}$, $\mathrm{SO}_{2}$ denoting the set of rotations in $\mathbf{R}^{2}$. Note that this is the typical situation for nonlinear elasticity for materials with different natural states (see [11]-[20]).

\section{NUMERICAL ESTIMATES}

From now on, for the sake of simplicity, we will restrict our analysis to problems of the type

$$
I=\inf _{W_{0}^{1, \infty}\left(\Omega, \mathbf{R}^{m}\right)} \int_{\Omega} \varphi(\nabla v(x)) d x
$$

where $\nabla v$ denotes the jacobian matrix of $v$ and $W_{0}^{1, \infty}\left(\Omega, \mathbf{R}^{m}\right)$ the set of Lipschitz continuous functions with values in $\mathbf{R}^{m}$, vanishing on the boundary of $\Omega$.

We denote by $\tau_{h}$ a regular triangulation of $\Omega$ and we set

$$
V_{h}^{0}=\left\{v: \Omega \rightarrow \mathbf{R}^{m}\left\{v \text { is continuous, } v=0 \text { on } \Gamma, v \text { is affine on each simplex of } \tau_{h}\right\} .\right.
$$

(The readers who are not familiar with the finite element method can assume $n=2$ or 3 , the element $K$ are then triangles or tetrahedrons splitting the domain $\Omega$ ). $V_{h}^{0}$ is the natural space to approximate $W_{0}^{1, \infty}\left(\Omega, \mathbf{R}^{m}\right)$ and the discrete version of $(2.1)$ is

$$
I_{h}=\inf _{V_{h}^{\circ}} \int_{\Omega} \varphi(\nabla v(x)) d x .
$$


We refer to [9], [11], [13], [14], [18] for various numerical computations regarding these probems. Note that one has clearly $I \leq I_{h}$. So, one is interested to obtain estimates for $I_{h}-I$ in terms of the mesh size $h=\max _{K \in \tau_{h}} h_{K}, h_{K}$ being the diameter of the element $K$.

One indirect outcome of such estimate is to produce a minimizing sequence. Indeed, assume that (2.1) does not admit a minimizer, i.e. there is no $u \in W_{0}^{1, \infty}\left(\Omega, \mathbf{R}^{m}\right)$ for which the infimum $I$ is achieved. If $v_{h} \in V_{h}^{0}$ is an element such that

$$
I_{h} \leq \int_{\Omega} \varphi\left(\nabla v_{h}(x)\right) d x \leq I_{h}+\epsilon_{h}
$$

and if

$$
0 \leq I_{h}-I \leq \delta_{h}
$$

then $\lim _{h \rightarrow 0} \epsilon_{h}=\lim _{h \rightarrow 0} \delta_{h}=0$ will imply that $v_{h}$ is a minimizing sequence. Conversely, we are not aware of any other method to get estimates of $I_{h}-I$ but by building up a $v_{h}$ such that (2.3) holds. Let us see the development of the above strategy on the simple example (1.4). We refer the interested reader to [4]-[10], for details on more complex situations.

So, let us prove :

Theorem 2.1: Assume that the triangulation of $\Omega$ is regular, i.e. the triangles are not allowed to flatten when the mesh size $h$ goes to 0 , then there is a constant $C$ such that

$$
I_{h}=\inf _{V_{h}^{0}} \int_{\Omega} v_{x}^{2}+\left(v_{y}^{2}-1\right)^{2} d x d y \leq C h^{\frac{1}{2}} .
$$

Proof : First note that there is no loss of generality in assuming $h<1$.

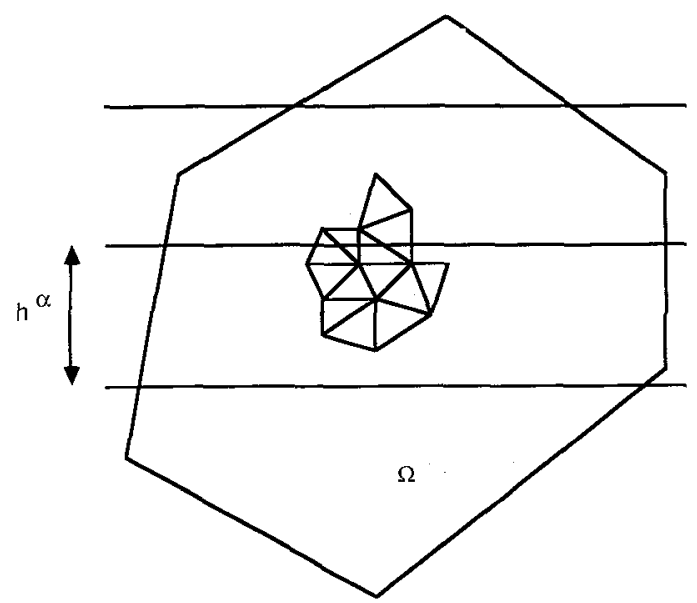

figure 1

Then, for any $\alpha \in(0,1)$ one has

$$
h^{\alpha}>>h
$$

when $h \rightarrow 0$. 
Let $\hat{u}_{h}$ be the function periodic of period $2 h^{\alpha}$ in the $y$ direction and defined by

$$
\hat{u}_{h}(x, y)=\left\{\begin{array}{c}
y \text { for } 0 \leq y \leq h^{\alpha} \\
-y+2 h^{\alpha} \text { for } h^{\alpha} \leq y \leq 2 h^{\alpha}
\end{array}\right.
$$

One has clearly

$$
\int_{\Omega}\left(\hat{u}_{h}\right)_{x}^{2}+\left(\left(\hat{u}_{h}\right)_{y}^{2}-1\right)^{2} d x d y=0
$$

However, $\hat{u}_{h} \notin V_{h}^{0}$ since the boundary conditions are not matched and also they are some triangles where $\hat{u}_{h}$ fails to be affine (see figure 1). In order to correct this one introduces

$$
\hat{u}_{h}^{\prime}=\hat{u}_{h} \wedge \operatorname{dist}(., \Gamma)
$$

where $\wedge$ denotes the minimum of two numbers, and $\operatorname{dist}(., \Gamma)$ the distance to the boundary $\Gamma$ of $\Omega$. Finally one sets

$$
u_{h}=\text { the interpolate of } \hat{u}_{h}^{\prime}
$$

i.e. the unique function of $V_{h}^{0}$ that agrees with $\hat{u}_{h}^{\prime}$ at the nodes of the triangulation. It is easy to check that

$$
0 \leq u_{h}, \hat{u}_{h}^{\prime}, \hat{u}_{h} \leq h^{\alpha}
$$

Moreover,

$$
\nabla u_{h}=W_{1} \quad \text { or } \quad W_{2}
$$

(see (1.5), (2.5)-(2.7)) except in a neighbourhood $N_{1}$ of $\Gamma$ where $u_{h}$ could be equal to dist(., $\Gamma$ ) and in a neighbourhood $N_{2}$ of thickness $2 h$ around the lines $y=k . h^{\alpha}, k \in \mathbf{Z}$ where interpolation takes place. If $|\Gamma|$ denotes the measure of $\Gamma$, i.e. its lengh, and $\mid$ the area of domains in $\mathbf{R}^{2}$ one has by (2.8)

$$
\left|N_{1}\right| \leq C|\Gamma| h^{\alpha}
$$

Moreover,

$$
\left|N_{2}\right| \leq 2 N h
$$

where $N$ is the number of horizontal lines $y=k \cdot h^{\alpha}$ cutting the domain $\Omega$ (see figure 1). If $D$ denotes the diameter of $\Omega$ one has

$$
(N-1) h^{\alpha} \leq D
$$

so that for $h<1$,

$$
N \leq C h^{-\alpha}
$$

for some constant $C$ so, for maybe an other constant $C$,

$$
\left|N_{2}\right| \leq C h^{1-\alpha}
$$

Since the triangulation $\tau_{h}$ has been assumed to be regular the gradient of $u_{h}$ remains uniformly bounded as well as the one of $\hat{u}_{h}^{\prime}$ and $\hat{u}_{h}$. So, for some constant $C$ one has

$$
\int_{\Omega}\left(u_{h}\right)_{x}^{2}+\left(\left(u_{h}\right)_{y}^{2}-1\right)^{2} d x d y \leq C\left\{\left|N_{1}\right|+\left|N_{2}\right|\right\} \leq C\left(h^{\alpha}+h^{1-\alpha}\right) \leq C h^{\alpha \wedge 1-\alpha} .
$$

Now, $\alpha \wedge 1-\alpha$ is minimum when $\alpha=1 / 2$ which gives (2.4). 
Remark 2.1: It is clear that (2.4) implies that the infimum of (1.4) is zero. Now, clearly this infimum is not achieved. Indeed, if for some $u$

$$
\int_{\Omega} u_{x}^{2}+\left(u_{y}^{2}-1\right)^{2} d x d y=0
$$

then, $u_{x}=0$ and since $u$ is vanishing on the boundary of $\Omega u \equiv 0$. But, obviously, $u=0$ does not satisfy (2.15).

Remark 2.2: This estimates is sharp (see [10]).

Remark 2.3: It follows from (2.8) that $u_{h} \rightarrow 0$ when $h \rightarrow 0$. In fact it can be shown that this is the behavior of any minimizing sequence. Moreover, we refer the interested reader to [5], [6] for a rigorous statement and justification, any minimizing sequence uses -as $u_{h}$ does- each of the wells with the same probability i.e. 1/2. In mechanical or physical terms it seems that a material with two natural states will switch infinitely many times between both of them in order to achieve a minimum of energy. It is an interesting domain of investigation to see if numerical simulations will produce oscillations similar to the ones detected in the theory that we have just briefly addressed above. This is the topic of our next section in a slightly more complex situation, yet very far from a realistic one.

Remark 2.4: Mathematically the coherence of the minimizing sequences is described through the notion of Young measure that has recently emerged in this kind of problems (see [1], [7], [15], [16], [22], [25]).

\section{COMPUTATIONS}

In the situation studied in the previous section the wells of the density of energy $\varphi$ were rank one compatible i.e. they were differing by a rank one matrix -in fact they were themselves rank one! In the situation where the wells $W_{i}$ (see (1.3)) are two by two rank one compatible one can get for (2.2) a result similar to the one of Theorem 2.1. (see [8]) and construct the minimizing sequences.

In order to analyse what is happening in the case where the wells are not rank one compatible we made in [9] some simulations in the case $m=n=2$. We took as wells the matrices (see [26])

$$
W_{1}=\left(\begin{array}{ll}
1 & 0 \\
0 & 2
\end{array}\right), W_{2}=\left(\begin{array}{cc}
2 & 0 \\
0 & -1
\end{array}\right), W_{3}=\left(\begin{array}{cc}
-1 & 0 \\
0 & -2
\end{array}\right) \quad, \quad W_{4}=\left(\begin{array}{cc}
-2 & 0 \\
0 & 1
\end{array}\right) .
$$

Clearly, when $i \neq j, W_{i}-W_{j}$ is not rank one. $\Omega$ being the unit square of $\mathbf{R}^{2}$, we considered the minimization problem

$$
\inf _{W_{0}^{1, \infty}\left(\Omega, \mathbf{R}^{2}\right)} \int_{\Omega} \varphi(\nabla v(x)) d x
$$

where $\nabla v$ denotes the jacobian matrix of $v$ i.e.

$$
\nabla v=\left(\begin{array}{ll}
\frac{\partial v^{1}}{\partial x_{1}} & \frac{\partial v^{1}}{\partial x_{2}} \\
\frac{\partial v^{2}}{\partial x_{1}} & \frac{\partial v^{2}}{\partial x_{2}}
\end{array}\right)
$$

and $W_{0}^{1, \infty}\left(\Omega, \mathbf{R}^{2}\right)$ the set of Lipschitz continuous functions with values in $\mathbf{R}^{2}$, vanishing on the boundary of $\Omega$. As energy density we took

$$
\varphi(W)=\prod_{k=1}^{4}\left\|W-W_{k}\right\|^{2}
$$


where \|\| denotes the usual euclidean norm of matrices i.e. $\|W\|^{2}=\operatorname{trace}\left(W^{T} W\right)$. First we have : Proposition 3.1: The problem (3.1) does not admit a minimizer i.e. the infimum (3.1) is not achieved.

Proof : One can show (see [9]) that the infimum (3.1) is zero. Then assume that for some $u \in$ $W_{0}^{1, \infty}\left(\Omega, \mathbf{R}^{2}\right)$

$$
\int_{\Omega} \varphi(\nabla u(x)) d x=0
$$

Then one has

$$
\varphi(\nabla u(x))=0 \quad \text { a.e. } x \in \Omega
$$

and thus

$$
\nabla u(x)=W_{i} \quad \text { a.e. } x \in \Omega
$$

In particular

$$
\frac{\partial u^{1}}{\partial x_{2}}=0 \quad, \quad \frac{\partial u^{2}}{\partial x_{1}}=0 \quad \text { a.e. } x \in \Omega
$$

So, $u^{1}$ is a function independent of $x_{2}$ and $u^{2}$ is a function independent of $x_{1}$. Since these two functions are vanishing on the boundary of $\Omega$ one has

$$
u^{1}=u^{2}=0
$$

i.e. $u=0$. But,

$$
\int_{\Omega} \varphi(0) d x=|\Omega| \varphi(0)>0
$$

Hence, a contradiction.

Denote by $\tau_{h}$ a triangulation of $\Omega$ and set

$$
v_{h}^{0}=\left\{v: \Omega \rightarrow \mathbf{R}^{2} \mid v \text { is continuous, } v=0 \text { on } \Gamma, v \text { is affine on each simplex of } \tau_{h}\right\} \text {. }
$$

Then the aproximate problem of (3.1) is

$$
\inf _{V_{h}^{0}} \int_{\Omega} \varphi(\nabla v(x)) d x
$$

Unlike the problem studied in the previous section we have been unable so far to construct a minimizing sequence for (3.8) and to get estimates similar to (2.4). It can also be shown that every minimizing sequence to (3.1) converges uniformly toward 0 and uses each of the wells $W_{i}$ with the same probability $1 / 4$ (see [9]). Unfortunately computers are still unable to find their way in constructing such "weird" minimizing sequences (see figure 2). 


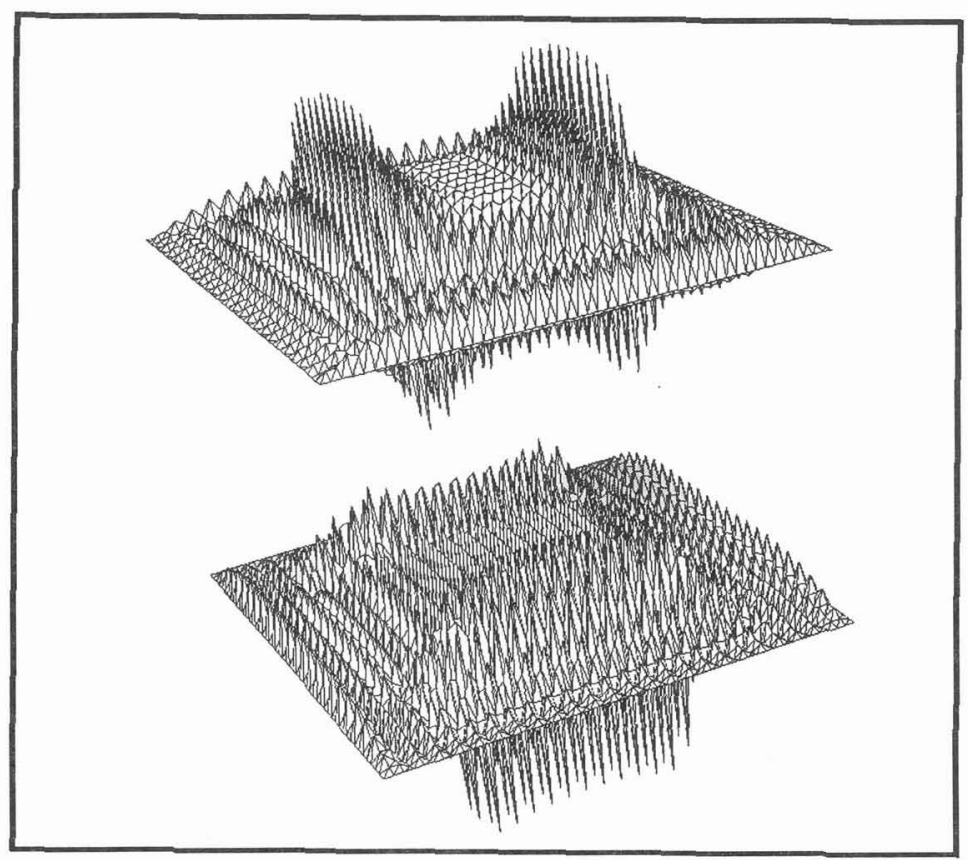

figure 2

We can show, however, that

$$
\lim _{h \rightarrow 0} \inf _{V_{h}^{0}} \int_{\Omega} \varphi(\nabla v(x)) d x=0 .
$$

Using annealing, random initial guesses and various numerical tricks we have been unable so far to get out of the local minima that we entcounter. Of course, due to the large number of problems of the type mentionned in section 1, one understand the relevance of attacking these questions. On the figure 2 -magnified due to the fact that minimizing sequences converge toward 0 - one can see the two components of a local minimum of the approximated problem (3.8).

Acknowledgements: The above picture has been realized by V. Lécuyer (see [9]) with the gOcad software and I would like to thank him and the gOcad team for their help.

\section{References}

[1] Ball J. M., A version of the fundamental theorem for Young measures, Partial Differential Equations and Continuum Models of Phase Transitions, Lecture Notes in Physics \# 344, M. Rascle, D. Serre and M. Slemrod, eds., Springer-Verlag, (1989), pp. 207-215.

[2] Ball J. M., James R. D., Arch. Rational Mech. Anal., 100, (1987), pp. 13-52.

[3] Ball J. M., James R. D., Phil. Trans. R. Soc. Lond. A, 338, (1992), pp. 389-450.

[4] Brighi B., Chipot M., SIAM J. Numerical Analysis, Vol 31, 1, (1994), pp. 128-148.

[5] Chipot M., Numerische Mathematik, 59, (1991), pp. 747-767.

[6] Chipot M., Collins C., SIAM J. of Numerical Analysis, 29, 4, (1992), pp. 1002-1019. 
[7] Chipot M., Kinderlehrer D., Arch. Rational Mech. Anal., 103, (1988), pp. 237-277.

[8] Chipot M., Collins C., Kinderlehrer D., Numerical Analysis of Oscillations in Multiple Well

Problems. Center for Nonlinear Analysis, Carnegie Mellon University (Preprint).

[9] Chipot M., Lécuyer V, Analysis and computations in the four-well problem, preprint \# 6, Center for Nonlinear Analysis, University of Metz (1994).

[10] Chipot M., Müller S., Sharp energy estimates to finite element approximation for nonconvex problems (to appear).

[11] Collins C., Thesis, University of Minnesota, 1990.

[12] Collins C., Kinderlehrer D., Luskin M., SIAM J. Numerical Analysis, Vol 28, (1994), pp. 321-332.

[13] Collins C., Luskin M., The computation of the austenitic-martensitic phase transition. Partial Differential Equations and Continuum Models of Phase Transitions, Lecture Notes in Physics \# 344, M. Rascle, D. Serre and M. Slemrod, eds., Springer-Verlag, Berlin, New York, (1989), pp. 34-50.

[14] Collins C., Luskin M., Computational results for phase transitions in shape memory materials, Smart Materials, Structures, and Mathematical Issues, C. Rogers, ed. Technomic Publishing Co., Lancaster, Pennsylvania, (1989), pp. 198-215.

[15] Dacorogna B., Direct methods in the calculus of variations, Springer Applied Math Sciences \# 78, (1988).

[16] Evans L. C., Weak Convergence Methods for Nonlinear Partial Differential Equations, A.M.S. Regional Conference Series in Mathematics \# 74, (1989).

[17] Fonseca I., J. Math. Pures et Appl., 67, (1988), pp. 175-195.

[18] Gremaud P. A., Numerical analysis of a nonconvex variational problem related to Solid-solid phase transitions, IMA Preprint Series, University of Minnesota, (1992).

[19] Gilbarg A. D., Trudinger N.S., Elliptic Partial Differential Equations of Second Order, Springer Verlag, (1985).

[20] James R. D., Kinderlehrer D., Theory of diffusionless phase transitions, Partial Differential Equations and Continuum Models of Phase Transitions, Lecture Notes in Physics \# 344, M. Rascle, D. Serre and M. Slemrod, eds., Springer-Verlag, Berlin, New York, (1989), pp. 51-84 [21] Kinderlehrer D., Remarks about equilibrium configurations of crystals, Material Instabilities in Continuum Mechanics and Related Problems, J. M. Ball ed., Oxford University Press, Oxford, (1987), pp. 217-242.

[22] Kinderlehrer D., Pedregal P., Characterization of gradient Young measures, Arch. Rat. Mech. Anal. 115, (1991), pp. 329-365.

[23] Kohn R., The relationship between linear and nonlinear variational models of coherent phase transitions, Proceedings of the Seventh Army Conference on Applied Mathematics and Computing, West Point, June 1989.

[24] Kohn R., S. Müller, Surface energy and microstructure in coherent phase transitions, Comm. Pure Appl. Math., 47, (1994), pp. 405-435.

[25] Tartar L., Compensated compactness and application to partial differential equations, Nonlinear analysis and mechanics: Heriot-Watt Symp IV, R.J. Knops ed., Pitman, (1979), pp. 136-212. [26] Tartar L., Some remarks on separately convex functions, Microstructure and phase transitions, D. Kinderlehrer et al. edts, Springer-Verlag, (1993), pp. 191-204. 